\title{
Rocas bajo el microscopio: acercamiento al estudio en lámina delgada de minerales y rocas
}

\author{
Patricia Acero Salazar ${ }^{1}$ Ester Mateo González ${ }^{2}$, Pedro Lucha López ${ }^{3}$ \\ ${ }^{1}$ Departamento de Ciencias de la Tierra. Facultad de Geología. 50009 Zaragoza. España. \\ patriace@unizar.es \\ ${ }^{2}$ Departamento de Didáctica de las Ciencias Experimentales. Facultad de Educación. 50009 Zaragoza. \\ España.emateog@unizar.es \\ ${ }^{3}$ Departamento de Didáctica de las Ciencias Experimentales. Facultad de Ciencias Humanas y de la \\ Educación.22003 Huesca.España.plucha@unizar.es
}

[Recibido en enero de 2013, aceptado en abril de 2013]

\begin{abstract}
En este trabajo se presenta una propuesta didáctica destinada a favorecer el acercamiento de los alumnos a una de las técnicas más habituales empleadas para el estudio de las rocas, sedimentos y minerales: la microscopía de luz polarizada. Para conseguir este objetivo, se proporciona a los alumnos un mínimo de conocimientos teóricos que les permite trabajar en grupos notablemente autónomos e identificar algunos de los componentes más frecuentes en distintos tipos de rocas y sedimentos. Con esta actividad, se familiariza a los estudiantes con algunas de las propiedades empleadas en microscopía óptica y con las propiedades de los propios componentes rocosos, y se proporcionan unas herramientas que facilitan el acercamiento a esta faceta del trabajo científico. Palabras clave: Microscopía de luz polarizada; Rocas; Minerales, Lámina delgada.
\end{abstract}

Rocks under the microscope: an approach to the study of thin sections of minerals and rocks

In this work, a didactic proposal is presented, aimed at favoring the contact of the students with one of the most usual techniques for the study of rocks, sediments and minerals: polarized light microscopy. With this purpose, a minimal set of theoretical basis is presented to the students, which allows them to work fairly autonomously and to identify some of the most frequent components in different rock and sediment types. By means of this activity, the students become familiar with some of the light properties and also with some of the mineral properties currently used in microscopy, encouraging their contact with this type of scientific work.

Keywords: Polarized light microscopy; rocks; minerals; thin sections.

\section{Introducción}

Los minerales constituyen un tema fundamental en cualquier nivel de enseñanza obligatoria, tanto por razones de tipo teórico-práctico como por razones didácticas. Son los materiales de los que está formada la parte inerte de la Tierra, los que soportan los diferentes procesos geológicos y también son la materia prima a partir de la cual se fabrican la mayoría de los objetos cotidianos. Por ello, constituyen un tema recurrente en la enseñanza en Primaria y en Secundaria. Trabajar con estos materiales, sus usos y su origen permite una aproximación a las Ciencias de la Tierra desde lo cotidiano y de forma global.

En esta actividad se proporciona a los alumnos un primer contacto con los procedimientos y fundamentos teóricos empleados para la identificación y estudio de minerales, rocas y sedimentos mediante el microscopio petrográfico. De esta manera, los estudiantes se enfrentan a una perspectiva nueva para ellos sobre las propiedades de la luz y de los propios minerales y, además, experimentan un acercamiento a esta faceta del trabajo científico del geólogo.

Aunque este tipo de trabajo con el microscopio petrográfico es habitual en los estudios universitarios relacionados con la Geología, su desarrollo con alumnos de enseñanza 
secundaria no lo es tanto. Esto se debe en ocasiones a la falta de equipamiento en los centros educativos pero también, en muchos casos, a la gran cantidad de tiempo requerida para que los alumnos puedan familiarizarse con los conceptos teóricos relacionados con la identificación de minerales y rocas. Para solventar en parte este último problema, en el diseño de actividad propuesto en este artículo, se aborda la identificación de minerales y otros componentes rocosos de forma directa, motivadora e intuitiva, basada en la simple observación y comparación con fotografías y en una sucinta explicación teórica sobre los fundamentos de la microscopía petrográfica. El carácter de iniciación de la actividad permite la participación con aprovechamiento en la misma, incluso de alumnos poco familiarizados con el microscopio de luz transmitida, la mineralogía y/o la petrografía. Esta actividad puede servir como punto de partida para iniciarles en el conocimiento de las características de las técnicas microscópicas y de las propiedades cristalinas y ópticas de minerales y rocas.

\section{Objetivos de la actividad propuesta}

Aunque se trata de una actividad de duración relativamente reducida (menos de 2 horas), con su realización se pueden alcanzar un buen número de objetivos, entre los cuales cabe destacar los siguientes:

1. Familiarizar a los alumnos con las técnicas microscópicas y con algunos de sus fundamentos, los cuales son aplicables en diversos campos científico-técnicos (ej. geología, biología, nanotecnología, ciencias médicas).

2. Fomentar algunas competencias características de la tarea científica como la observación, comparación, descripción e identificación de minerales y otros componentes de sedimentos y rocas.

3. Ofrecer una nueva perspectiva sobre propiedades y características de los minerales y rocas que son poco evidentes a simple vista y acercar a los alumnos una de las herramientas que utilizan los profesionales o investigadores de la geología para llevar a cabo sus proyectos.

4. Favorecer la capacidad para el trabajo autónomo y el cuidado y respeto por el material de laboratorio.

\section{Fundamento teórico para la actividad}

La actividad propuesta se basa en el uso de microscopía de luz polarizada para la identificación de distintos componentes de rocas y sedimentos. Esta técnica se basa en la observación y el reconocimiento de las distintas propiedades ópticas que tienen los diferentes minerales y otros componentes de las rocas y sedimentos cuando la luz polarizada los atraviesa (Carretero y Pozo 2007).

Se denomina luz polarizada a aquella en la que todas las ondas vibran en paralelo a un mismo plano formado por su trayectoria y la dirección de vibración. Este tipo de luz es indistinguible a simple vista de la luz normal, en la que las ondas vibran en múltiples direcciones del espacio. Una de las formas de obtener luz polarizada, que es la usada comúnmente en los microscopios petrográficos, es utilizar un filtro al que se denomina polarizador o nícol.

$\mathrm{Si}$ interponemos en la trayectoria de un haz de luz polarizada un segundo filtro polarizador (denominado analizador), éste sólo será atravesado completamente por dicho haz si las direcciones de los planos de vibración de ambos polarizadores coinciden (lo que se denomina luz polarizada no analizada o, más comúnmente, posición de nícoles paralelos). Por el contrario, si vamos girando el analizador con respecto a esta posición de coincidencia, la cantidad de ondas capaz de atravesar ambos filtros será cada vez menor, hasta llegar a ser 
completamente nula cuando las direcciones de los planos de vibración de ambos filtros polarizadores sean perpendiculares (luz polarizada analizada o posición de nícoles cruzados), tal como se muestra en la figura 1. En ausencia de una lámina o grano mineral entre ambos nícoles, no habrá ninguna onda de luz que alcance el ocular y se tendrá oscuridad total. Sin embargo, si interponemos entre los dos filtros polarizadores una lámina mineral lo suficientemente fina como para dejar pasar la luz, dicha lámina provocará una serie de fenómenos de interferencia entre las ondas polarizadas que la atraviesan. Debido a estos fenómenos, algunas de las ondas que atraviesan los minerales de la lámina observada sí estarán alineadas con la dirección del analizador, por lo que lo atravesarán, alcanzarán el ocular y el observador verá luz y color (figura 1).

Estos fenómenos son de enorme importancia para la observación de minerales en el microscopio petrográfico, ya que permiten la observación de algunas propiedades ópticas características de cada mineral concreto y que permiten diferenciar a unos de otros (MacKenzie y Adams 1997). Algunas de estas propiedades (ej. relieve) se observan normalmente con nícoles paralelos, mientras que otras (ej. color de interferencia) se deben observar con nícoles cruzados. Por lo tanto, una de las operaciones más habituales durante la observación mineral en el microscopio petrográfico consiste en alternar el uso de un solo nícol o dos nícoles cruzados.

Para la observación de estas propiedades ópticas es necesario disponer de granos minerales lo suficientemente finos como para permitir pasar la luz a su través o, más comúnmente, de finas secciones de rocas u otros materiales, obtenidas por corte y pulido (Ques y Tritlla 1997). Estas secciones se denominan generalmente láminas delgadas y suelen tener un grosor de unos 0,03 $\mathrm{mm}$ y unas dimensiones de unos $5 \mathrm{~cm} \times 3 \mathrm{~cm}$.

A lo largo de la actividad propuesta se trabaja precisamente con láminas delgadas de distintos tipos de rocas, escogidos de forma que la identificación de sus minerales y otros componentes sea relativamente sencilla y pueda ser llevada a cabo con unas nociones mínimas de óptica mineral.

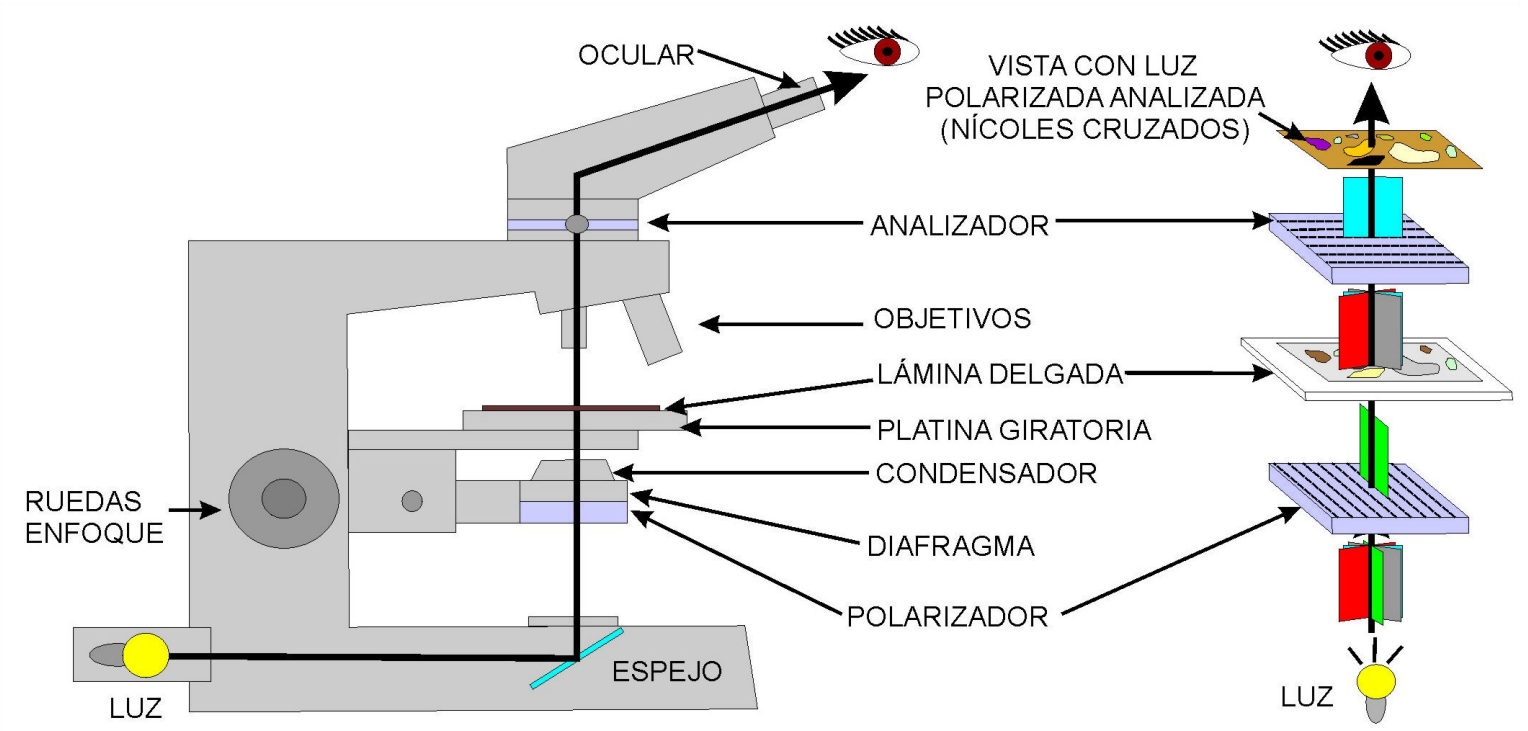

Figura 1. Esquema de los principales elementos en el microscopio de luz polarizada y relación de cada uno de ellos con las propiedades de la luz y con la observación de láminas delgadas de minerales, rocas y otros materiales. 


\section{Contexto general de la actividad}

Según nuestra experiencia, puede ser realizada satisfactoriamente con grupos de hasta 20 participantes por cada tutor de actividad. El tiempo estimado para la realización de esta experiencia es de alrededor de 1 hora y media, incluyendo el tiempo necesario para organizarse inicialmente y para que cada alumno recoja el material empleado al finalizar la actividad.

\section{Diseño de la actividad}

\section{Equipamiento necesario}

El equipamiento básico necesario consiste en los siguientes elementos:

- Microscopio de luz polarizada o petrográfico, en el que se puedan observar las propiedades minerales tanto con nícoles paralelos como con nícoles cruzados. Aunque la actividad se podría realizar con un único microscopio para todo el grupo, lo ideal es disponer de un equipo para cada pareja de estudiantes.

- Varias colecciones de láminas delgadas de dos o tres tipos de roca en las que aparezcan distintos minerales y otros componentes habituales en las rocas (cuarzo, feldespatos, micas, calcita, olivino, vidrio volcánico, fósiles, etc). Para facilitar la identificación, se debe intentar que las rocas seleccionadas sean claramente muy distintas y se intentará que el número de componentes de roca con el que se trabaja no sea muy elevado (no más de 6-8 elementos). En nuestro caso, la actividad suele realizarse con una roca carbonatada con abundantes fósiles, con una roca volcánica y con una roca plutónica.

- Fichas de identificación mineral, en las que aparezcan distintas fotografías del aspecto que ofrecen típicamente cada uno de los minerales con los que se trabajará tanto en observación con nícoles paralelos como en nícoles cruzados (véase una ficha de ejemplo en la figura 2A). Cada grupo de alumnos dispone de una de estas fichas al lado del microscopio en el que está trabajando. Es muy importante para el éxito de la actividad que los minerales y componentes de roca seleccionados presenten características ópticas claramente diagnósticas y que éstas aparezcan correctamente reflejadas en las fichas de identificación.

- Fichas (en la figura 2B se muestra un ejemplo) en las que los participantes deben dibujar esquemas de lo observado en cada lámina y anotar los minerales identificados y otras observaciones (tamaño, bordes cristalinos, presencia de impurezas o inclusiones, etc). Idealmente, cada alumno debe disponer de una ficha para cada tipo de roca a observar, de forma que los esquemas y anotaciones sean de tipo individual aunque el trabajo en el microscopio se realice en grupo.

\section{Secuenciación de la actividad}

La actividad se organiza en cuatro partes, siendo las dos primeras de carácter teórico-práctico y las dos siguientes de tipo básicamente práctico. Para mantener el interés de los alumnos es conveniente que la duración combinada de las dos partes iniciales no exceda los 45 minutos y que durante éstas se fomente la participación de los alumnos mediante preguntas, cadenas deductivas y manipulación del material a emplear.

1) Introducción sobre la actividad profesional del geólogo y sobre los métodos de estudio de las rocas, describiendo el proceso seguido desde la recogida de la muestra de roca en campo hasta la observación en el microscopio de luz trasmitida. Opcionalmente, se puede hacer circular entre los alumnos un juego de material similar al mostrado en la figura 3 (o, en su defecto, la propia fotografía), en el que se incluyen muestras de roca correspondientes a los cuatro pasos básicos desde la roca en campo hasta la elaboración de la lámina delgada. De esta 
forma, los alumnos perciben que el trabajo con láminas delgadas de roca no es algo abstracto y alejado de la realidad sino una forma útil de preparación. Duración máxima aconsejada: 10-15 minutos.

\section{ASI SON EN EL MICROSCOPIO}

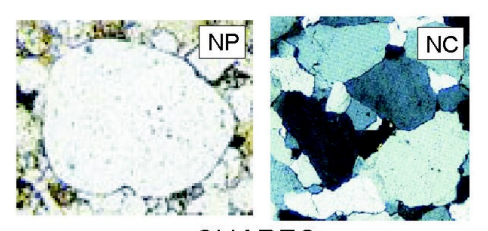

CUARZO

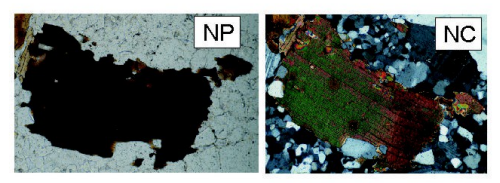

MICA (BIOTITA)

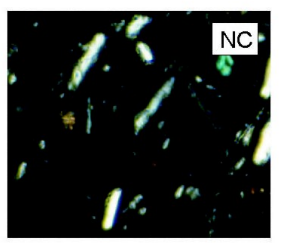

VIDRIO

(con Plagioclasas y Olivinos)

A $\mathrm{NP}$ Con nícoles paralelos

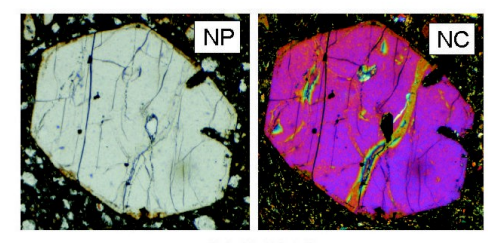

OLIVINO

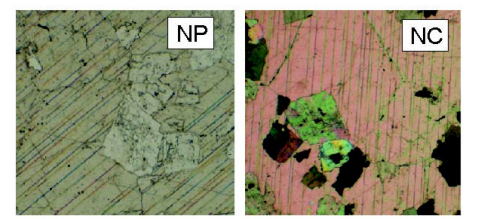

CALCITA

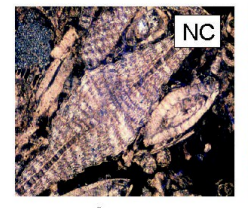

FÓSILES

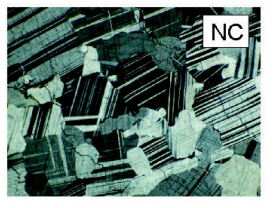

PLAGIOCLASA

NC Con nícoles cruzados
Dibuja un esquema de lo que ves y marca en el cuadro con una cruz los componentes de roca que reconozcas

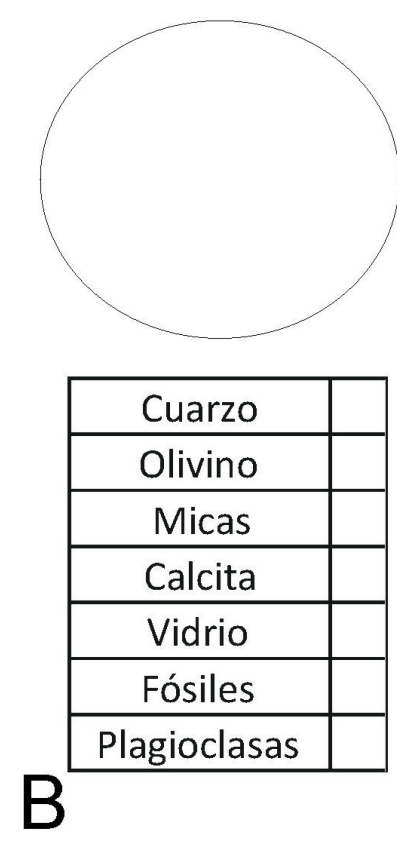

Figura 2. Material de apoyo empleado en el desarrollo de la actividad: (A) modelo de fichas de identificación mineral mediante fotografías de sus propiedades ópticas diagnósticas con nícoles paralelos y cruzados; (B) modelo de fichas en las que los estudiantes deben realizar un esquema y señalar los minerales identificados en cada una de las láminas delgadas incluidas en la actividad.

2) Explicación breve de los conceptos teóricos necesarios para desarrollar la actividad. Duración máxima aconsejada: 30 minutos. Es conveniente comenzar esta parte de la sesión con una breve descripción (unos 10 minutos) de las propiedades de la luz polarizada y del uso de nícoles paralelos y cruzados para el estudio de la materia cristalina, similar a la expuesta en la sección dedicada al fundamento teórico de la actividad. A continuación, se exponen (durante unos 10 minutos) las principales propiedades diagnósticas de cada mineral o componente de roca a observar en lámina delgada, tanto con nícoles paralelos como con nícoles cruzados, enfatizando especialmente aquellas que más los diferencian entre sí. Finalmente, se realiza una descripción del funcionamiento del microscopio de luz trasmitida (unos 10 minutos). En ésta última parte resulta muy interesante que los estudiantes vayan manipulando los distintos componentes a emplear a medida que se realiza la explicación, lo que facilita su comprensión.

3) Trabajo autónomo de los participantes con supervisión del profesor o tutor de la actividad. Duración aconsejada: al menos 30 minutos. A lo largo de esta parte de la sesión, cada grupo de estudiantes trabaja en un microscopio observando las distintas láminas delgadas de roca puestas a su disposición. En paralelo a la observación y con la ayuda de las fichas de identificación y sus fotografías (figura 3A), cada participante debe realizar en las fichas (figura 3B) un esquema sencillo de cada una de las láminas observadas, intentando identificar los 
minerales y otros componentes de roca que aparecen en la misma. Para ello, los participantes de cada grupo deben confrontar sus opiniones e intentar llegar a un acuerdo.

Durante esta parte de la actividad, es fundamental que ésta se encuentre supervisada por el tutor de la misma, de forma que las dudas y problemas surgidos (en general relacionados con el manejo del microscopio o con dificultades para diferenciar entre varios minerales) puedan ser resueltos de forma rápida y eficaz.

4) Puesta en común. Duración aproximada: 15 minutos. En esta parte de la sesión, se realiza una puesta en común de los resultados obtenidos por cada pareja o grupo de estudiantes y el tutor indica qué componentes aparecen en cada una de las láminas delgadas observadas. Dicha puesta en común se puede realizar en forma de concurso, de manera que los aciertos y fallos de cada grupo al identificar los distintos componentes de cada lámina delgada sean contabilizados y conduzcan a uno o varios ganadores.

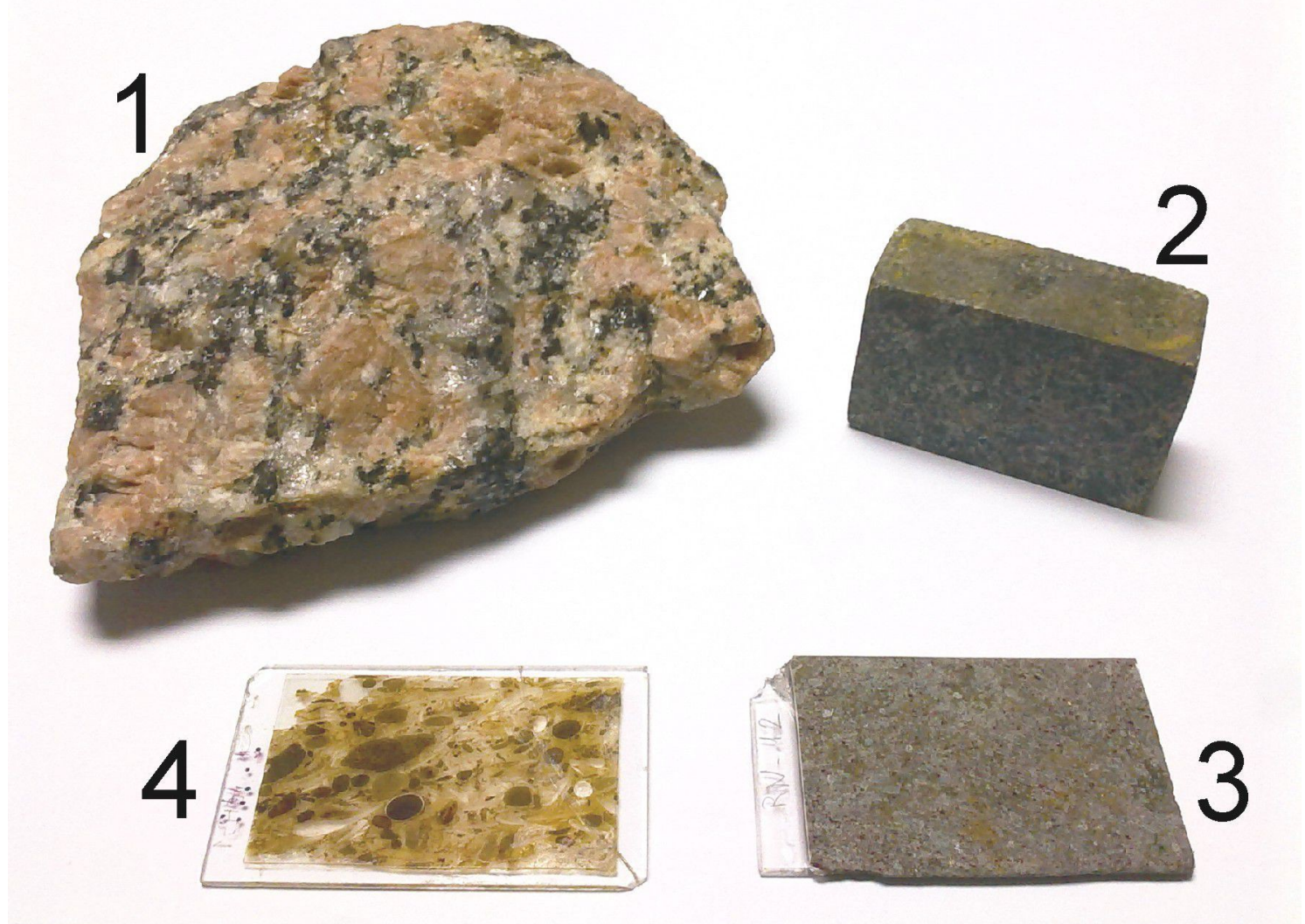

Figura 3. Material empleado para mostrar a los alumnos los pasos básicos desde la recogida de una muestra de roca en campo (1) hasta la elaboración de una lámina delgada para su observación en el microscopio petrográfico (4), pasando por distintas etapas de corte y pulido (2 y 3).

\section{Comentarios finales y valoración de la actividad}

Esta actividad se ha llevado a cabo con muy buenos resultados en distintos eventos de divulgación científica desarrollados en el Departamento de Ciencias de la Tierra de la Universidad de Zaragoza (Jornadas de Puertas Abiertas, Campus Científicos de Verano y Semanas de Inmersión en Ciencias). Los participantes han sido generalmente estudiantes de los últimos cursos de la Enseñanza Secundaria Obligatoria (ESO) y de Bachillerato, con edades comprendidas entre los 15 y los 18 años. 
Los participantes en la actividad en sus distintas ediciones han valorado muy positivamente la misma y sus reacciones habituales son de sorpresa, especialmente ante la observación de minerales en lámina delgada con luz paralela analizada, y de entusiasmo, al ser capaces de reconocer y diferenciar distintos minerales tras recibir únicamente algunas indicaciones básicas. Es destacable además la buena capacidad de manejo de los componentes básicos del microscopio petrográfico que manifiestan la mayor parte de los alumnos al final de la actividad.

En muchos casos, la realización de la práctica ha estimulado la participación y la curiosidad de los alumnos participantes, que se muestran interesados en recibir explicaciones adicionales sobre las propiedades y minerales que observan. Por este motivo, consideramos que esta actividad puede constituir un excelente punto de partida para tratar temas relacionados con las técnicas de estudio geológico, con la materia cristalina y sus propiedades y con distintos aspectos de los minerales, rocas y sedimentos.

En nuestra opinión, la actividad podría ser fácilmente realizable en cualquier otra Facultad de Geología y en centros de enseñanza secundaria en los que el material requerido esté disponible, como complemento práctico a las asignaturas de Ciencias de la Naturaleza, Geología o similares.

\section{Referencias}

Carretero M. I., Pozo M. (2007) Mineralogía aplicada: salud y medio ambiente. Madrid. Thomson.

MacKenzie W. S., Adams A. E. (1997) Atlas en color de rocas y minerales en lámina delgada. Barcelona. Masson.

Ques J., Tritlla J. (1997) Introducción a las técnicas de preparación de láminas delgadas, pp. 33-37 en Atlas de Asociaciones Minerales en Lámina Delgada, J. C. Melgarejo (ed.). Barcelona. Publicaciones de la Universidad de Barcelona y Fundació Folch. 